\title{
Obsessive compulsive disorder and migraine: case report, diagnosis and therapeutic approach
}

\author{
Luiz Paulo Bastos Vasconcelos $\cdot$ Melissa Costa Silva $\cdot$ Esther Angélica Coelho Costa \\ Ariovaldo Alberto da Silva Júnior · Rodrigo Santiago Gómez · Antônio Lúcio Teixeira
}

Received: 23 May 2008/Accepted: 31 August 2008/Published online: 19 September 2008

(C) Springer-Verlag 2008

\begin{abstract}
Psychiatric disorders, notably mood and anxiety disorders, are frequently associated with migraine and chronic daily headaches. The obsessive-compulsive disorder (OCD) is included in the spectrum of anxiety disorders and may be a comorbid condition in headache patients. However, little information has been reported in the literature about this association. This is an important issue as OCD may contribute to the development or maintenance of treatment-resistant chronic headaches. In this paper, we describe a young female patient with refractory chronic migraine and OCD. Considerations on diagnosis, management and treatment of these comorbid conditions are presented.
\end{abstract}

Keywords Migraine - Chronic daily headache . Obsessive-compulsive disorder · Psychiatric comorbidity

\section{Introduction}

Psychiatric disorders are frequently found in migraine. Mood and anxiety disorders are the psychiatric

L. P. B. Vasconcelos - M. C. Silva - E. A. C. Costa

A. A. da Silva Júnior · R. S. Gómez · A. L. Teixeira Headache Clinic, University Hospital, School of Medicine, Federal University of Minas Gerais (UFMG),

Belo Horizonte, Brazil

\author{
A. L. Teixeira $(\square)$ \\ Departamento de Clínica Médica, Faculdade de Medicina, \\ Federal University of Minas Gerais (UFMG), \\ Av. Alfredo Balena, 190. Santa Efigênia, Belo Horizonte, \\ MG 30130-100, Brazil \\ e-mail: altexr@gmail.com
}

comorbidities most described in these patients [1-3]. The literature also indicates that psychiatric comorbidity is even higher in transformed migraine and chronic daily headaches, particularly in the presence of analgesic abuse [2]. The connection between psychiatric disorders and headache syndromes is not fully understood, but this issue has been extensively debated in the literature. Regardless of the mechanism of comorbidity, psychiatric conditions may influence patient's outcome and selection of headache treatment [1-3].

It is worth mentioning that the bulk of this knowledge derives from studies performed with an adult migraine population. Some studies also investigated psychiatric and behavioral problems in childhood and adolescence [4-6]. For instance, Guidetti et al. [4] have found that anxiety and depression symptoms were associated with migraine in children and adolescents, but not with tension-type headaches, a finding that is in line with studies in adults. Notably, there is also some support in the literature to suggest that medication overuse and psychiatric comorbidity are risk factors for the development of chronic headaches in adolescents, as occurs in adult population [7, 8]. By contrast, other studies showed that, despite behavioral symptomatology in children with headaches, most of these symptoms were subclinical and did not qualify children for psychiatric diagnosis $[5,6]$. Furthermore, similar levels of behavioral and emotional symptoms were reported in young tension-type headache and migraine patients [5].

Obsessive-compulsive disorder (OCD) belongs to the anxiety spectrum disorders and may be a comorbid condition in patients with migraine. OCD seems to occur at higher frequency in patients with migraine than in the general population, but little information regarding this association is available [9]. 
In this paper, we report a young female patient with migraine presenting also a comorbid OCD. This association had relevant implications for the headache treatment and prognosis which are discussed.

\section{Case report}

A 14-year-old white female patient was referred to our service to be evaluated for a severe daily headache which was refractory to diverse non-steroidal anti-inflammatory drugs (NSAIDs), triptans and ergotamine. In her first evaluation, the patient reported a disabling daily headache with frequent episodes of exacerbation started 1 year before. These episodes were characterized by frontal throbbing headaches along with nausea, vomiting, photophobia and phonophobia. Such exacerbation episodes used to occur 10 days per month and could not be relieved with any analgesic medication. The patient habitually abused NSAIDs and triptans in a daily pattern to relieve pain and avoid exacerbations of headache. Headache episodes started when she was 12 years old and became more frequent in the year before consultation.

On examination, the patient demonstrated excessive worry about her condition and gave a detailed description of her pain. She also reported that she could not "stop thinking" about the possibility of a severe disease leading to her pain and described that she was "continuously worried and alert" to the emergence of severe crisis of headache. In addition, she reported intrusive thoughts about the fear of doing harm to others as a consequence of her acts that lead to mental compulsions. She also exhibited worries about symmetry. She had experienced these symptoms as mildly distressful since she was 10 years old. Her neurological examination was unrevealing as well as neuroimaging and routine laboratory tests. Thus, according to the reviewed criteria of the ICHD II [10], the patient fulfilled the criteria for chronic migraine in association to analgesic medication overuse.

The patient and her mother were informed about the possible nature of the headache and that analgesic overuse could have impact on its evolution. A weaning treatment with corticosteroids, restricted use of analgesics and headache prophylaxis with flunarizine were prescribed. Flunarizine was chosen as the patient had not been treated before with a single-drug regimen for migraine prophylaxis and is the treatment of choice for migraine prophylaxis [11]. A structured pain calendar was offered to the patient, so she could make a detailed description of her headache and indicate the days of severe pain during 1 month period.

After 6 weeks the patient returned with a complex headache report along with a series of charts. She reiterated the obsessive thoughts about her pain. Besides, the daily use of acute relief medications was clearly a way to attenuate excessive worries and the recurrent negative thoughts about her condition. No significant improvement of headache was observed. She was interviewed by a psychiatrist who performed the diagnosis of OCD according to DSM-IV criteria [12] based on a structured interview (Mini International Neuropsychiatric Interview, M.I.N.I.) [13]. A neuropsychological evaluation, including Rey Auditory Verbal Learning test, Stroop test, verbal fluency test and Trail Making test (parts A and B), was also performed. She exhibited only an increased time of execution of Trail Making test's part B, a task aimed at measuring visual attention and concentration. This result indicates abnormality in the mental processing speed for executing tasks which require the ability to make alternate conceptual changes (require also the ability to maintain alert states) as has been previously reported for young migraineurs [14].

Despite the institution of cognitive-behavior therapy for OCD and the maintenance of the drug therapy for migraine, the patient evolved with poor clinical response and persistence of headache. After 6 months of follow-up, with no significant improvement, she quit our service and her treatment was discontinued.

\section{Discussion}

The connection between psychiatric disorders and migraine and/or $\mathrm{CDH}$ has been extensively described in the literature $[1-3,9,15]$. The incidence of psychiatric disorders may reach up to $90 \%$ of patients with $\mathrm{CDH}$ [9, 16]. A great body of evidence even suggests that the adequate treatment of psychiatric comorbidity determines a significant improvement of the quality of life of headache patients $[3,9,17-20]$.

The association between migraine and/or $\mathrm{CDH}$ and psychiatric disorders seems to be not merely incidental [9, 17]. There is much debate on the possible causal relationship between migraine and psychiatric disorders [15]. Many authors believe that there is a common physiopathological pathway for both entities in which the serotoninergic system may be involved [1-3].

The OCD is characterized by intrusive and recurrent obsessive thoughts and repetitive behaviors that are engaged to relieve the anxiety caused by the obsessive thoughts. The OCD is classified under the group of anxiety disorders and its association with migraine and/or CDH has been also described $[9,15,17,18]$. It is worth mentioning that patients with migraine are five times more susceptible to suffer from OCD [9, 16].

In the present case report, our patient exhibited chronic migraine with analgesic medication overuse and OCD. She was refractory to any treatment proposed. In this context, 
many factors may be involved in therapeutic failure and some considerations must be done. First of all, the lack of adhesion to treatment and the failed attempts to withdrawn analgesic overuse may be considered relevant factors of therapeutic failure. It is already defined that analgesic overuse is important to establish and maintain chronic headaches [21]. Furthermore, the presence of obsessive thoughts and her compulsion to take analgesics suggest that OCD may be a contributing condition to the chronic and refractory migraine in this patient. Anxiety disorders may play an important role in peripheral and central mechanisms of pain sensitization which contributes to the evolution to chronic headaches $[18,19]$. The headache sensitization becomes even more evident if we consider that patients with psychiatric comorbidity usually overuse analgesics or other relief medications at a higher frequency. Besides medication overuse, $40 \%$ of these patients do not return to consultations and $50 \%$ do not take prescribed headache prophylactic drugs [17].

The management of patients with headache and psychiatric comorbidity is quite difficult and there are no established protocols to treat both conditions simultaneously. Merely prescribing a medication without demanding an active participation of the patient may lead to frustrating results. The establishment of a good doctorpatient relationship and the education of the patients and their relatives are of extreme importance for a successful therapy $[9,17-20]$. The combination of drug therapy and psychotherapy may be a very effective approach for OCD and migraine. Regarding the relevant role of social and familiar contexts in children's and adolescents' headaches, family counseling may be recommended for increasing compliance to treatment [7].

In respect to the drug therapy, it may be started with a conventional migraine treatment which includes immediate relief medications and prophylactic drugs. Withdrawal from medication overuse when clinically present must always be attempted. Beta blockers, flunarizine and topiramate must be used with caution because they can worsen psychiatric symptoms $[3,18,19]$. There is even one single case report of OCD induced by topiramate [22]. However, controlled studies are necessary to confirm this observation. Valproate could be an option for chronic migraine prophylaxis, but it should be used with caution in female adolescents taking into account its significant potential to induce weight gain. The serotonin selective reuptake inhibitors (SSRIs) antidepressants are effective to treat OCD, but they are not effective in migraine treatment $[3$, 18] and may even exacerbate it [20]. Besides that, SSRIs must be used with caution in children and adolescents since many undesirable side effects may appear such as suicidal behavior [23]. Clormipramine may be effective for both headache and OCD and can be indicated in such cases [20].
There is considerable evidence suggesting a role for atypical antipsychotics, such as olanzapine, in augmentation therapy for OCD and there have been several anecdotal reports of their efficacy in the management of refractory migraine [24].

We decided to use flunarizine in the present case as it presents great efficiency and safeness in the prophylaxis of children and adolescents with migraine and the risks for exacerbating obsessive-compulsive symptoms are very low $[11,25]$. The cognitive-behavioral therapy (CBT) is considered one-first line treatment of $\mathrm{OCD}$, notably in children and adolescents [17, 26]. CBT is based on cognitive restructuration and patient education for solving problems. The active participation of the patient in CBT is of great value in improving adhesion and efficacy of the concomitant treatment for headache and psychiatric comorbidity [19]. Despite the institution of a combined therapy and the special attention offered by our team, the patient maintained poor response to treatment. Maybe this could be explained by the lack of adherence to the treatment. On follow-up the patient frequently missed sessions of psychotherapy and many times resisted to use flunarizine. It is worth mentioning that even when careful integrative approach is employed with patients with headache and psychiatric comorbidity, unfavorable outcomes may occur [17].

As the association between headache and psychiatric disorders seems to be frequent and impact on the outcome, it is essential to look for psychiatric symptoms in all patients with headache, notably those with refractory to treatment $[9,16]$. Simple questions about sadness symptoms, unwillingness, irritability and anxiety and the observation of clinical signs such as obsessive thoughts must be a routine. Some screening instruments, such as PRIME-MD, were designed to detect psychiatric comorbidity in patients in the general practice. They are more frequently used by researches, but they might be used and incorporated in the headache clinics [16].

In conclusion, OCD must be considered as a factor contributing to headache treatment resistance. Its screening, as in the other psychiatric disorders, is of extreme importance for the better management and treatment of headache patients.

Conflict of interest None.

\section{References}

1. Juang KD, Wang SJ, Fuh JL et al (2000) Comorbity of depression and anxiety disorders in chronic daily headache and its subtypes. Headache 40:818-823

2. Radat F, Swendsen J (2005) Psychiatric comorbidy in migraine: a review. Cephalalgia 25:165-178 
3. Torelli P, Lambru G, Manzoni GC (2006) Psychiatric comorbidity and headache: clinical and therapeutical aspects. Neurol Sci 27:S73-S76

4. Guidetti V, Galli FT, Fabrizi P et al (1998) Headache and psychiatric comorbidity: clinical aspects and outcomes in an 8-year follow-up study. Cephalalgia 18:455-462

5. Mazzone L, Vitiello B, Incorpora G, Mazzone D (2005) Behavioural and temperamental characteristics of children and adolescents suffering from primary headache. Cephalalgia 26:194-201

6. Pakalnis A, Gibson J, Colvin A (2005) Comorbidity of psychiatric and behavioral disorders in pediatric migraine. Headache 45:590-596

7. Pakalnis A, Butz C, Splaingard D, Kring D, Fong J (2007) Emotional problems and prevalence of medication overuse in pediatric chronic daily headache. J Child Neurol 22:1356-1359

8. Wang SJ, Fuh JL, Lu SR, Juang KD (2007) Outcomes and predictors of chronic daily headache in adolescents: a 2-year longitudinal study. Neurology 68:591-596

9. Baskin SM, Lipchik GL, Smitherman TA (2006) Mood and anxiety disorders in chronic headache. Headache 46(Suppl 3): S76-S87

10. Headache Classification Subcommittee of the IHS (2004) The International Classification of Headache Disorders, 2nd edn. Cephalalgia 24 (Suppl 1):8-160

11. Lewis D, Ashwal S, Hershey A et al (2004) Practice parameter: pharmacological treatment of migraine headache in children and adolescents: report of the American Academy of Neurology Quality Standards Subcommittee and the Practice Committee of the Child Neurology Society. Neurology 63(12):2215-2224

12. American Psychiatric Association (2000) Diagnostic and statistical manual of mental disorders, 4th edn. American Psychiatric Association, Washington, DC

13. Sheehan DV, Lecrubier Y, Sheehan KH, Amorim P, Janavs J, Weiller E, Hergueta T, Baker R, Dunbar GC (1998) The MiniInternational Neuropsychiatric Interview (M.I.N.I.): the development and validation of a structured diagnostic psychiatric interview for DSM-IV and ICD-10. J Clin Psychiatry 59(Suppl 20): 22-33

14. Riva D, Aggio F, Vago C et al (2006) Cognitive and behavioural effects of migraine in childhood and adolescence. Cephalalgia 26:596-603
15. Sareen J, Jacobi F, Cox BJ et al (2006) Disability and poor quality of life associated with comorbid anxiety disorders and physical conditions. Arch Intern Med 166:2109-2116

16. Maizels M, Smitherman TA, Penzien DB (2006) A review of screening tools for psychiatric comorbidity in headache patients. Headache 46(Suppl 3):S98-S109

17. Baskin SM (2007) Managing the "difficult" headache patient. Neurol Sci 28:S78-S83

18. Savarese M, Guazzelli M, Prudenzano MP et al (2005) Tertiary treatment for psychiatric comorbidity in headache patients. J Headache Pain 6:231-233

19. Lipchik GL, Smitherman TA, Penzien DB et al (2006) Basic principles and techniques of cognitive-behavioral therapies for comorbid psychiatric symptoms among headache patients. Headache 46(Suppl 3):S119-S132

20. Griffith JL, Razavi M (2006) Pharmacological management of mood and anxiety disorders in headache patients. Headache 46(Suppl 3):S133-S141

21. Bigal ME, Rapoport AM, Sheftell FD et al (2004) Transformed migraine and medication overuse in a tertiary headache centerclinical characteristics and treatment outcomes. Cephalalgia 24:483-490

22. Thuile J, Even C, Guelfi JD (2006) Topiramate may induce obsessive-compulsive disorder. Psychiatry Clin Neurosci 60(3):394

23. Cohen D (2007) Should the use of selective serotonin reuptake inhibitors in child and adolescent depression be banned? Psychother Psychosom 76:5-14

24. Silberstein SD, Peres MF, Hopkins MM et al (2002) Olanzapine in the treatment of refractory migraine and chronic daily headache. Headache 42:515-518

25. Boccia G, Del Giucce E, Crrisanti AF et al (2006) Functional gastrointestinal disorders in migrainous children: efficacy of flunarizine. Cephalalgia 26(10):1214-1219

26. O'Kearney RT, Anstey KJ, Von Sanden C (2006) Behavioural and cognitive behavioural therapy for obsessive compulsive disorder in children and adolescents. Cochrane Database Syst Rev 18(4):CD004856 